\title{
Tumor neuroectodérmico primitivo do terceiro ventrículo associado a metástase leptomeníngea
}

\section{Primitive Neuroectodermal Tumor of the Third Ventricle Associated with Leptomeningeal Metastases}

\author{
Jacques Lara-Reyna ${ }^{1}$ Natalia Burgos-Morales ${ }^{1}$ José Rodriguez-Matias ${ }^{2}$ David Martínez-Neira ${ }^{2}$ \\ Bolívar Cárdenas-Mera ${ }^{2}$
}

${ }^{1}$ Médico Residente do Serviço de Neurocirurgia, Hospital Luis Vernaza, Guayaquil, Equador

2 Médico Tratante do Serviço de Neurocirurgia, Hospital Luis Vernaza, Guayaquil, Equador

\begin{abstract}
Address for correspondence Natalia Burgos, MD, Departamento de Neurocirurgia. Hospital Luis Vernaza. Loja 700 y Escobedo, Guayaquil, Equador (e-mail: nati_burgosm@hotmail.com).
\end{abstract}

Arq Bras Neurocir 2015;34:157-161.

\section{Resumo \\ Palavras-Chave \\ - tumores neuroectodérmicos primitivos \\ - PNET \\ - carcinomatose meníngea}

Abstract
Keywords
- primitive
neuroectodermal
tumors
- PNET
- leptomeningeal
carcinomatosis

Os tumores neuroectodérmicos primitivos (PNET) são tumores malignos, não diferenciados, raramente apresentados na idade adulta, principalmente os de localização supratentorial. Apresentamos neste artigo o caso de um paciente de 19 anos, que nos chegou transferido de outro hospital com o diagnóstico de hidrocefalia. A existência do PNET associado a carcinomatose leptomeníngea foi comprovada como causa da hidrocefalia.

Primitive neuroectodermal tumors (PNET) are malignant tumors exceptionally present in adulthood, especially those with supratentorial location. In this article, we present the case of a young man who was transfer from another institution with the diagnosis of hydrocephalus; during his stay, we corroborated the presence of supratentorial PNET associated to leptomeningeal carcinomatosis that was a cause of hydrocephalus.

\section{Introdução}

Os PNET constituem um grupo heterogêneo de neoplasias formadas por células não diferenciadas ou pobremente diferenciadas durante o desenvolvimento embrionário do sistema nervoso. Estes tumores têm comportamento clini- camente agressivo, sendo catalogados no grau IV da classificação da OMS para tumores cerebrais. Os PNET do sistema nervoso central (SNC) abarcam um espectro de lesões neoplásicas observadas principalmente em crianças e jovens e têm como principal exemplo ao meduloblastoma, localizado de maneira clássica na fossa posterior. Os PNET são
Recebido

August 25, 2014

Aprovado

March 31, 2015
DOI http://dx.doi.org/ 10.1055/s-0035-1554046. ISSN 0103-5355.
Copyright $(2015$ by Thieme Publicações License terms Ltda, Rio de Janeiro, Brazil 
extremadamente infrequentes nos adultos, representando menos do $0,5 \%$ de todos os tumores intracranianos. ${ }^{1-4}$

\section{Exposição do Caso}

Paciente masculino de 19 anos, sem antecedentes patológicos conhecidos, que acudiu após apresentar quadro clínico de três meses de evolução caracterizado por cefaleia holocraniana pulsátil, vômitos, alterações de conduta, desorientação temporoespacial e movimentos tônico-clônicos generalizados. No exame físico encontrou-se diplopia esquerda pela paralisia do sexto nervo craniano. Realizou-se tomografia computadorizada e depois ressonância magnética nuclear (RMN) do cérebro nas quais pode-se observar hidrocefalia obstrutiva supratentorial, discreto aumento do tamanho da hipófise, lesão tumoral no terceiro ventrículo e notório reforço leptomeníngeo com pequenos micronódulos projetados sobre as cisternas basais, cerebelosas e periependimárias (-Figs. 1 e 2). Os resultados das provas de hormônios foram normais (TSH 1,80; prolactina 7,7; LH 3,0; FSH 2,5; testosterona 3,59; cortisol plasmático 15,4; ACTH 15,3; T3 0,75; FT4 1,86). Não houve achados relevantes na tomografia computadorizada de tórax e abdome.

Colocou-se um sistema de derivação ventriculoperitoneal, obtendo líquido cefalorraquidiano e observando pressão de abertura elevada; esta intervenção aliviou totalmente os sintomas do paciente. A análise do líquido cefalorraquidiano mostrou hiperproteinorraquia $(60 \mathrm{mg} / \mathrm{dL})$, incremento da celularidade (glóbulos brancos $5,3 / \mathrm{mm}^{3}$ ) e hipoglicorraquia (35 mg/dL). As provas para TB (ADN-PCR), as técnicas de coloração de Gram, de Ziehl-Neelsen e o cultivo foram negativos.

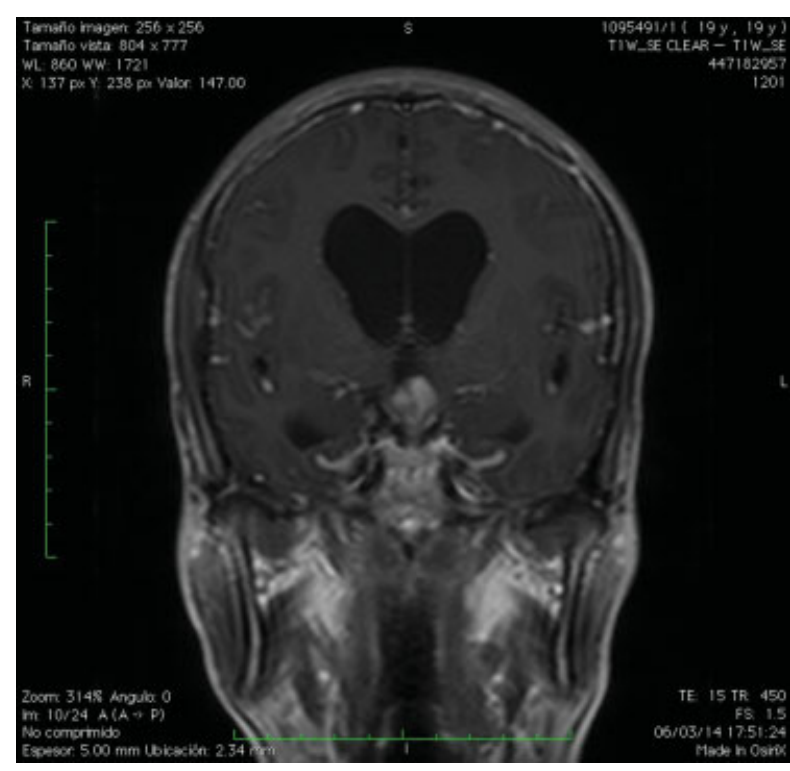

Fig. 1 imagem coronal de ressonância magnética onde se pode evidenciar uma lesão tumoral no terceiro ventrículo captante de contraste.

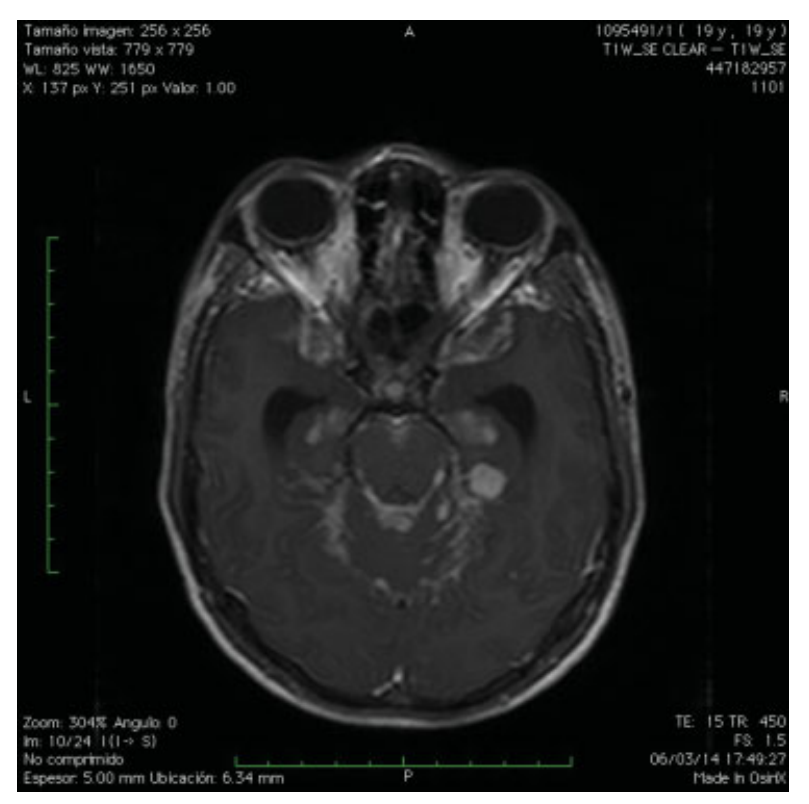

Fig. 2 Imagem axial de ressonância magnética com contraste, onde se evidencia marcado reforço leptomeníngeo y presença de pequenas micronodulações nas cisternas basais.

Realizou-se uma craniotomia em que se observou aracnoidite basal e um tumor vascularizado no terceiro ventrículo. Os achados histopatológicos foram fragmentos da neoplasia constituída por células pequenas com núcleos arredondados e poligonais, núcleos hipercromáticos com atividade mitótica aumentada, dispostos de maneira difusa sobre um estroma fibrovascular. A técnica de imuno-histoquímica foi positiva para sinaptofisina, CD99, neurofisina e Ki-67, compatíveis com tumor neuroectodérmico primitivo (-Fig. 3).

\section{Discussão}

Os sPNET (tumores neuroectodérmicos primitivos supratentoriais) são tumores embrionários malignos, não diferenciados ou pobremente diferenciados, localizados no sistema nervoso central de maneira extracerebelar.

Foram descritos pela primeira vez em 1973 por Hart e Earle como tumores morfologicamente similares aos meduloblastomas, mas de localização externa ao cerebelo. Recentemente se soube que o prognóstico e a genética molecular são diferentes entre os sPNET e os PNET infratentorias, e que eles provavelmente deveriam ser classificados em separado dos meduloblastomas ${ }^{1-3,5-7}$ (-Tabela $\mathbf{1}$ ).

Na idade adulta, os sPNET representam menos do $0,5 \%$ de todos os tumores intracranianos. Até agora, menos de cem casos foram documentados. Segundo a revisão da literatura de Papadopoulos, foram reportados 70 casos de sPNET. Dos 21 casos reportados na série de Balafuta, somente um caso teve localização intraventricular. Além disso, cinco casos de sPNET intraventriculares foram achados na própria revisão da literatura. Estes fatos fazem relevante a apresentação de nosso caso de sPNET localizado no terceiro ventrículo. ${ }^{6,8,12}$ 


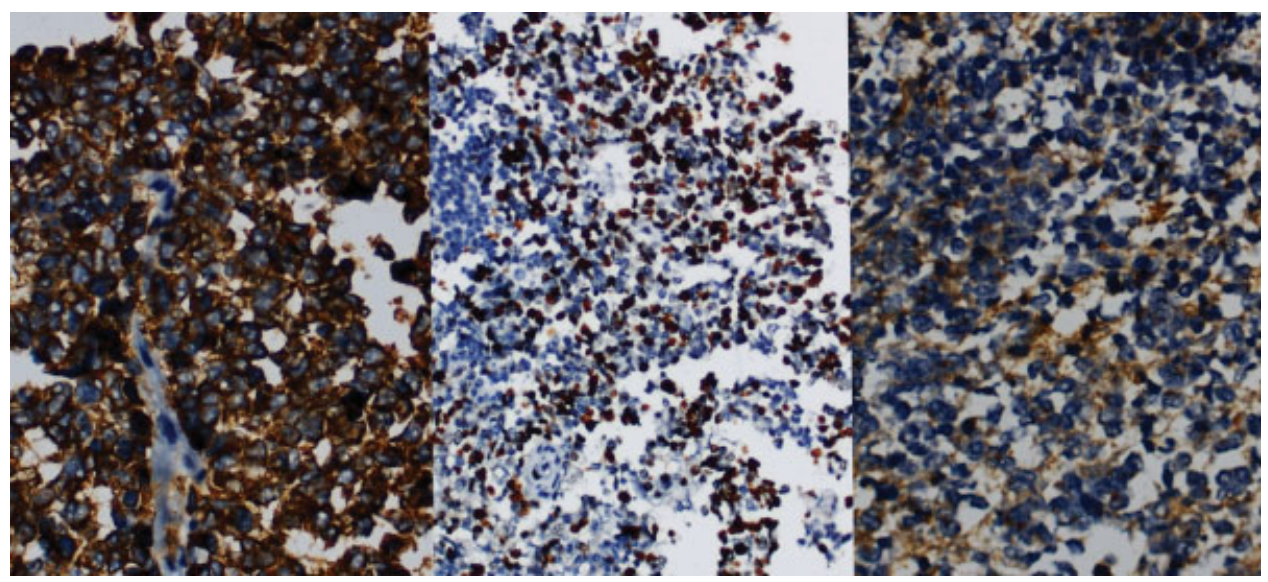

Fig. 3 Imunohistoquímica positiva para sinaptofisina (magnificação 40x), KI 67 (magnificação 10x) e CD99 (magnificação 40x), compatíveis com tumor neuroectodérmico primitivo.

A apresentação clínica dos sPNET é inespecífica, na maioria dos casos os sintomas estão relacionados com o aumento da pressão intracraniana, como nos tumores supratentoriais; em outros casos estão relacionados com a obstrução da circulação do líquido cefalorraquidiano dentro do sistema ventricular. Entre os sintomas estão cefaleia, vômitos, alterações da conduta, papiledema, oftalmoplegia. Nos tumores da fossa posterior, podem-se agregar outros sintomas como ataxia, vertigem e nistagmo. ${ }^{1,4,6,13}$

A ressonância magnética é superior para definir os limites e a localização exata da lesão, no diagnóstico radiológico. Na sequência ponderada em T1 pode existir hipossinal heterogêneo que pode aumentar de acordo com a presença de componente hemático intratumoral. Na sequência ponderada em T2 se aprecia um isossinal em relação ao parênquima normal, mas ela pode-se apresentar com marcado hipersinal. Uma característica importante na sequência contrastada com gadolínio é a disseminação leptomeníngea que ocorre em aproximadamente $20 \%$ dos sPNET. 1,6,14,15

Na histologia, os tumores neuroectodérmicos primários supratentoriais apresentam células neuroepiteliais primitivas não diferenciadas ou pobremente diferenciadas, com alta relação núcleo/citoplasma, núcleos basófilos arredondados ricos em cromatina, os quais são sinal de cariorrexe ou apoptose. Podem-se observar as pseudorrosetas de HornerWright, nas quais as células neoplásicas lançam curtos prolongamentos para o centro anuclear da roseta; a presença destas pseudorrosetas é típica, mas não é essencial para o diagnóstico. ${ }^{1}$

As técnicas de imuno-histoquímica podem ser usadas para identificar os componentes pobremente diferenciados das células neoplásicas dos sPNET. As células tumorais podem ser reativas a sinaptofisina, neuroenolase específica

Tabela 1 Comparação entre os meduloblastoma e os SPNET

\begin{tabular}{|l|l|l|}
\hline & sPNET & Meduloblastoma \\
\hline Prevalência & $\begin{array}{l}<1 \% \text { dos tumores } \\
\text { pediátricos do SNC }\end{array}$ & $\begin{array}{l}\text { Cerca de 20\% dos } \\
\text { tumores pediátricos do SNC }\end{array}$ \\
\hline Idade média de apresentação & 3 anos & 5 anos \\
\hline Sexo & Masculino = Feminino & Masculino $>$ Feminino \\
\hline Imagens & Reforço heterogêneo & Reforço homogêneo \\
\hline Histopatologia & $\begin{array}{l}\text { Pobremente diferenciado, } \\
\text { células neuroepiteliais, núcleos } \\
\text { arredondados pequenos }\end{array}$ & Igual \\
\hline Biologia molecular & $\begin{array}{l}\text { Sem anomalias cromossômicas } \\
\text { específicas }\end{array}$ & Deleção 17p, inserção 17q \\
\hline Metástase & 5 a 39\% & 40 a 60\% \\
\hline Tratamento & $\begin{array}{l}\text { Cirurgia + irradiação } \\
\text { cranioespinal + quimioterapia }\end{array}$ & Igual \\
\hline Sobrevivência aos 3 anos livre de progressão & 45 a 47\% & $>80 \%$ \\
\hline
\end{tabular}

Fonte: Tonn et al. ${ }^{1}$ 
(NSE), proteína glial fibrilar ácida (GFAP) e CD99. Os achados citogenéticos são relativamente escassos, os SPNET não mostram anomalias cromossômicas específicas além da deleção de $14 q$ em quatro de cada dez casos. ${ }^{1,16}$

Quase 5\% dos pacientes com câncer desenvolvem complicações associadas com a disseminação leptomeníngea. De 5 a $10 \%$ dos pacientes com carcinomatose leptomeníngea apresentam-na como primeira manifestação de uma patologia oncológica concomitante. ${ }^{1,6,13,14}$

A carcinomatose meníngea se caracteriza pela invasão de células tumorais no líquido cefalorraquidiano e nas meninges, seja por tumores primários do SNC ou por tumores sólidos (rabdomiossarcoma, neuroblastoma, meduloblastoma), linfoma ou leucemia, principalmente nos estados avançados da enfermidade. A presença de disseminação leptomeníngea nos tumores primários do SNC é de 1 a 32\% de acordo com a a etiologia histológica, idade do paciente e doenças concomitantes. ${ }^{13-15}$

Os sintomas são muito variados nestes casos e dependem do grau de disseminação e das estruturas próximas. As mais evidentes são mencionadas na $\boldsymbol{- T a b e l a ~} \mathbf{2}$.

O diagnóstico dessa condição é feito por dados radiológicos e estudo do líquido cefalorraquidiano (LCR). A presença de células tumorais no LCR é determinante para o diagnóstico de disseminação tumoral leptomeníngea, mas em algumas séries aproximadamente $50 \%$ das avaliações não mostraram sua presença, precisando-se novas punções tanto lombares como ventriculares. ${ }^{13,14,17}$

As características mais importantes do LCR nesses casos são uma pressão da abertura maior a $150 \mathrm{H} 20$, hiperproteinorraquia ( $>50 \mathrm{mg} / \mathrm{dL}$ ), incremento da celularidade (glóbulos brancos $\left.>4 / \mathrm{mm}^{3}\right)$ e hipoglicorraquia $(<40 \mathrm{mg} / \mathrm{dL})^{13}$

Os dados de neuroimagen são primordiais para determinar infiltração meníngea. A ressonância magnética com gadolínio tem mais sensibilidade e especificidade do que a tomografia e do que a ressonância sem contraste. Os achados mais frequentes são realce cisternal, ependimário, subepen- dimário e tentorial de maneira irregular, nódulos subaracnóideos e interventriculares com destacado realce e dilatação ventricular. ${ }^{13,15,17}$

O tratamento dos SPNET é multidisciplinar. A primeira linha terapêutica consiste na resseção tumoral e tem como objetivos o controle eficiente da patologia, obter amostra do tumor para a pesquisa histopatológica e melhorar os sintomas do paciente. Nesses tumores com limites mal definidos, o uso das técnicas de neuronavegação na abordagem cirúrgica tem um papel importante, assim como a colocação de sistemas de derivação ventriculares externos ou permanentes nos casos de hidrocefalia. ${ }^{1-3}$

Depois da resseção cirúrgica, o passo seguinte é a irradiação do eixo cranioespinal em pacientes com mais de 3 anos de idade. Na maioria dos pacientes diagnosticados com sPNET, a radioterapia no leito cirúrgico do tumor, assim como no eixo cranioespinal, é um coadjuvante necessário dada a relativa radiossensibilidade das células embrionárias. Não existe consenso definitivo sobre a dose da radiação efetiva para o tratamento dos sPNET, mas o regime estandardizado é de 4.680 a 6.000 cGy no sítio primário e de 2.700 a 4.000 cGy para o eixo cranioespinal. ${ }^{1}$

Em relação à quimioterapia, atualmente não existe um tratamento coadjuvante padronizado, mas o uso do regime associado de vincristina com nitrosourea combinado com prednisona ou cisplatino tem obtido uma sobrevida ao redor de $45 \%$ em 3 anos. ${ }^{1-4}$

O prognóstico dos pacientes com sPNET é mau, com alto risco de recorrência e disseminação leptomeníngea, tendo comportamento mais agressivo e menos quimiossensível que os meduloblastomas. Com uma abordagem cirúrgica adequada, radioterapia e quimioterapia, a taxa de sobrevivência aos 3 anos é de 45 a 47\%. Têm sido reportados indícios de melhores resultados com o uso de quimioterapia com células-mães, mas ainda não temos dados precisos e fidedignos que os corroborem. ${ }^{1-4,6,7}$

Tabela 2 Signos e sintomas da carcinomatose leptomeníngea por localização e em ordem decrescente de frequência

\begin{tabular}{|c|c|c|}
\hline & Sintoma & Signos \\
\hline Hemisfério cerebral & $\begin{array}{l}\text { Cefaleia } \\
\text { Alteração do estado mental } \\
\text { Náuseas/vômitos } \\
\text { Debilidade do hemicorpo } \\
\text { Convulsões }\end{array}$ & $\begin{array}{l}\text { Alteração do estado mental } \\
\text { Convulsões } \\
\text { Papiledema } \\
\text { Debilidade focal } \\
\text { Hemiparesia }\end{array}$ \\
\hline Nervos craniais & $\begin{array}{l}\text { Diplopia } \\
\text { Perda auditiva } \\
\text { Intumescimento facial } \\
\text { Diminuição da acuidade visual }\end{array}$ & $\begin{array}{l}\text { Oftalmoplegia (VI, III, IV) } \\
\text { Debilidade facial (VII) } \\
\text { Diminuição da acuidade auditiva (VIII) } \\
\text { Neuropatia facial (V) } \\
\text { Debilidade do hipoglosso (XII) }\end{array}$ \\
\hline Medula espinhal e raízes nervosas & $\begin{array}{l}\text { Debilidade nas extremidades } \\
\text { Dor } \\
\text { Instabilidade da marcha } \\
\text { Disfunção vesical } \\
\text { Disfunção intestinal }\end{array}$ & $\begin{array}{l}\text { Debilidade nas extremidades } \\
\text { Perda da sensibilidade (dermátomos/segmentos) } \\
\text { Reflexos tendinosos profundos anormais } \\
\text { Rigidez da nuca (meningismo) } \\
\text { Ataxia da marcha } \\
\text { Dor na elevação da perna estendida }\end{array}$ \\
\hline
\end{tabular}

Fonte: Bomgaars L et al. ${ }^{13}$ 


\section{Conclusão}

Os tumores primitivos neuroectodérmicos supratentoriais são tumores com comportamento agressivo e elevada mortalidade para os quais atualmente não existe um enfoque multimodal específico. Seu estudo abarca uma grande variedade de situações diagnósticas e terapêuticas. A resseção tumoral completa acompanhada de radioterapia e quimioterapia é o objetivo primário para tentar incrementar a sobrevida em pacientes portadores dessa neoplasia. Um diagnóstico adequado radiológico e de laboratório, assim como a busca do tumor primário nos casos de apresentação com disseminação leptomeníngea, em um período de tempo relativamente curto, poderia ajudar a programar estratégias adequadas de tratamento.

\section{Referências}

1 Tonn JC, Westphal M, Rutka JT, Grossman SA. Neuro-Oncology of CNS Tumors. 1st ed. Heidelberg: Springer; 2006

2 Quiñones-Hinojosa A. Schmidek \& Sweet. Operative Neurosurgical Technique, Indication, Methods and Results. 6th ed. Philadelphia: Elsevier Sanders; 2012

3 Winn R. Youmans Neurological Surgery. 6th ed. Philadelphia: Elsevier Sanders; 2011

4 Hart MN, Earle KM. Primitive neuroectodermal tumors of the brain in children. Cancer 1973;32(4):890-897

5 Shingu T, Kagawa T, Kimura Y, Takada D, Moritake K, Hoshii Y. Supratentorial primitive neuroectodermal tumor in an aged patient -case report-. Neurol Med Chir (Tokyo) 2005;45(10):530-535
6 DePaiva T, Pimenta S, Santos G, Souza Dias JE, Ayer TE, Oliveira E. Supratentorial primary neuroectodermal tumor (PNET) inside the third ventricle in adult: a rare case report. Arq Bras Neurocir 2013;32(2):125-129

7 Louis DN, Ohgaki H, Wiestler OD, et al. The 2007 WHO classification of tumours of the central nervous system. Acta Neuropathol 2007;114(2):97-109

8 Papadopoulos EK, Fountas KN, Brotis AG, Paterakis KN. A supratentorial primitive neuroectodermal tumor presenting with intracranial hemorrhage in a 42-year-old man: a case report and review of the literature. J Med Case Reports 2013;7:86-91

9 Balafouta MJ, Kouvaris JR, Miliadou AC, et al. Primitive neuroectodermal tumour in a 60-year-old man: a case report and literature review. Br J Radiol 2003;76(901):62-65

10 Davis PC, Wichman RD, Takei Y, Hoffman JC Jr. Primary cerebral neuroblastoma: CT and MR findings in 12 cases. AJR Am J Roentgenol 1990;154(4):831-836

11 Pearl GS, Takei Y, Bakay RA, Davis P. Intraventricular primary cerebral neuroblastoma in adults: report of three cases. Neurosurgery 1985;16(6):847-849

12 Lawandy S, Hariri OR, Miulli DE, et al. Supratentorial primitive neuroectodermal tumor in an adult: a case report and review of the literature. J Med Case Reports 2012;6:361-364

13 Levin V, Bernard W. Cancer in Nervous System. 2nd ed. New York: Oxford University Press; 2002

14 Baiges-Octavio JJ, Huerta-Villanueva M. Meningeal carcinomatosis. Rev Neurol 2000;31(12):1237-1241

15 Orrison W. Neurorradiología. 1era ed. Madrid: Harcourt; 2001

16 Girschick HJ, Klein R, Scheurlen WG, Kühl J. Cytogenetic and histopathologic studies of congenital supratentorial primitive neuroectodermal tumors: a case report. Pathol Oncol Res 2001; 7(1):67-71

17 Krygier G, Roldan G, Gaggero F, Mañana G, Levin R. Carcinomatosis meníngea en tumores solidos. Arch Med 2000;4:147-154 